Check for updates

Cite this: RSC Adv., 2019, 9, 34772

Received 24th September 2019 Accepted 19th October 2019

DOI: $10.1039 / c 9 r a 07745 f$

rsc.li/rsc-advances

\section{Convenient synthesis of uncovered imprinted microspheres by Ganoderma lucidum spore- stabilized pickering emulsion polymerization and their enhanced recognition of spiramycin $\dagger$}

\author{
Yanzhuo Zhu, ab Honghui Teng, ${ }^{c}$ Dongshu Sun, ${ }^{c}$ Dayu Jiang ${ }^{\text {ab }}$ \\ and Yongsheng Yan (iD *a
}

In this work, a new and simple method was developed for the synthesis of uncovered and high-selectivity spiramycin-based molecularly imprinted microspheres (SP-MIMs) by Pickering emulsion polymerization using spiramycin as templates. And surprisingly the solid particles were absent on the surface of imprinted microspheres, which can be ascribed to the Ganoderma lucidum spores (GLS): they were firstly selected to be the stabilizers for the Pickering emulsion in this new strategy. Through a series of adsorption experiments, the uncovered SP-MIMs were proven to possess more excellent selective recognition and removal ability for template SP in aqueous solution. And SP-MIMs could be reused for many times without significant loss of adsorption capacity, indicating the satisfactory regeneration performance. Therefore, SP-MIMs can be employed as a promising adsorbent for the selective removal of SP from aqueous media, and this strategy will contribute to overcoming the shortcomings of stabilizer particles on the surface of the as-prepared imprinting microspheres, because these solid particles lack the special selective recognition activity of template molecules, and may disturb the adsorption effect of imprinting microspheres.

\section{Introduction}

In recent years, many kinds of organic pollutants, including antibiotics, have been introduced into the aquatic environment. ${ }^{1,2}$ For instance, spiramycin (SP), a kind of typical 16membered ring macrolides antibiotic, has been detected in Italian aquatic environment. ${ }^{3}$ The presence of antibiotics in bodies of water around the world has drawn very special attention because of the potential promotion role towards the breeding of drug-resistant bacteria. ${ }^{4}$ Thus, it is very important to monitor, recognize and remove the antibiotic residues in water in time. Molecularly imprinted polymers (MIPs) have tailored recognition sites with high affinity towards given target molecules, ${ }^{5}$ so they have been widely applied in selective separation and recognition of many different targets, such as bifenthrin, ${ }^{6}$ lambda-cyhalothrin, ${ }^{7}$ tetracycline, ${ }^{8}$ protein ${ }^{9}$ and so on. To date the MIPs can be synthesized using various

${ }^{a}$ Key Laboratory of Preparation and Applications of Environmental Friendly Materials (Jilin Normal University), Ministry of Education, Changchun, 130103, China. E-mail: jsdxyan@126.com

${ }^{b}$ College of Chemistry, Jilin Normal University, Siping, 136000, China

${ }^{c}$ College of Environmental Science and Engineering, Jilin Normal University, Siping, 136000, China

$\dagger$ Electronic supplementary information (ESI) available. See DOI: 10.1039/c9ra07745f techniques, such as bulk polymerization, precipitation polymerization or emulsion polymerization, etc. Among the many polymerization approaches, Pickering emulsion polymerization has gained more and more attention in recent years, because it has the advantages of simplicity, high yields of polymer and good control of final particle size, which make it be suitable for preparing the desired MIPs. ${ }^{10}$

The type of Pickering emulsion mainly includes the oil-inwater $(\mathrm{o} / \mathrm{w})$ and water-in-oil (w/o), which is believed to be determined in some way by the particle wettability. ${ }^{\mathbf{1 1}}$ And the wettability of particles, expressed in terms of the contact angle $\theta$ of a particle at the interface of oil and water, is a major factor to determine the surface activity of the particles and the type of Pickering emulsion. ${ }^{12}$ When the value of $\theta$ of particles is measured to be beyond $90^{\circ}$, particles are hydrophobic and can be used to stabilize w/o emulsions. Whereas when the measured value of $\theta$ is below $90^{\circ}$, particles are usually hydrophilic and can stabilize o/w emulsions. If the particles can be completely wetted by water or oil, they will remain dispersed in either phase and can not be used to stabilize Pickering emulsions. ${ }^{12}$ Based on this, the diverse solid particles have been developed to be used as emulsion stabilizers, such as polymer, ${ }^{2}$ magnetic particles, ${ }^{13}$ silica, ${ }^{14} \mathrm{TiO}_{2},{ }^{15}$ clays ${ }^{16}$ and isocyanate microcapsules. ${ }^{17}$ However, most of the known inorganic particles have been found to be inherently hydrophilic and thus their 
surfaces need to be modified. For example, the satisfactory wettability of particles was obtained by the adsorption of amphipathic emulsifiers, such as small molecular weight charged surfactants and oleic acid. ${ }^{18}$ Besides, many kinds of polymers for the appropriate emulsion stabilizers are prepared., ${ }^{2,19,20}$ However, it is noticeable that the surface of MIPs via Pickering emulsion polymerization are covered with a layer of stabilizer particles, which have no affinity binding sites for the template molecules. Instead, they may interfere with the rebinding of imprinted sites to template molecules.

As a species of basidiomycotina mushroom, Ganoderma lucidum (GL) is a famous traditional Chinese medicine. Ganoderma lucidum spores (GLS) are generative cells with a protective bilayer sporoderm structure, which produced by the sporophore. There are many active ingredients in GLS, including ergosterol, triterpenoids, unsaturated fatty acids, and polysaccharides, ${ }^{21}$ and so GLS have strong bioactivity and have been widely used in antitumor, modulating immunity, protecting liver and so on. ${ }^{22}$ The spheroid GLS are thermally and mechanically stable, and the sizes of GLS particles are about $6.5-8 \times 9.6-12.6 \mu \mathrm{m} .{ }^{23}$ It is interesting that the GLS have a large internal space and thus they will be a promising biomaterial. ${ }^{24}$ However, to date, there are no reports on the synthesis of MIPs using the GLS-stabilized Pickering emulsion polymerization.

In this work, for the first time, we report the utilization of GLS as stabilizers of Pickering emulsion and the synthesis of a novel type of uncovered spiramycin-based molecularly imprinted microspheres (SP-MIMs). Most interestingly and importantly, because GLS can be easily separated from the surface of imprinted microspheres in water after the polymerization, there was not a layer of GLS on the surface of the prepared imprinted microspheres. However, the absence of GLS on the surface of MIPs may be benefit for the extraction and rebinding of template molecules. In addition, the selective adsorption and recognition behaviors toward template molecules (SP) were investigated in detail. And the prepared imprinted microspheres were also characterized by some techniques, such as scanning electron microscopy (SEM), Fourier Transform Infrared spectra (FTIR) and so on. The results showed that the prepared SP-MIMs possessed good thermal stability, large specific surface, perfect surface morphology and excellent selectivity to template molecules.

\section{Materials and methods}

\subsection{Materials}

Ganoderma lucidum spores (GLS) was obtained from Changbai Mountain Protection Development Zone Senbao speciaity store (Jilin, China). Spiramycin (SP) were supplied by $\mathrm{Hu}$ Bei Zhiqi biochemical Co., Ltd. (Hubei, China). EM, azithromycin (AZM) and chloromycetin (CHL) were purchased from Hu Bei Hengshuo chemical co., Ltd. Ethanol, toluene, acetic acid, methanol and phosphoric acid (85\%) were received from Sinopharm Chemical Reagent Co., Ltd. (Shanghai, China). 2,2'-Azobis(2methylpropionitrile) (AIBN) was obtained from Tianjin Guangfu Fine Chemical Reagent Co.,Ltd. Ethyleneglycol dimethacrylate (EGDMA) and methacrylic acid (MAA) were all received from Aladdin Reagent Co., Ltd. (Shanghai, China). Deionized water was used in all experiments.

\subsection{Instrument}

Fourier Transform Infrared (FTIR) spectra were recorded on a Nicolet Nexus-470 FTIR apparatus (USA) using KBr pellet in the range of $4000-400 \mathrm{~cm}^{-1}$. The morphology of SP-MIMs was examined by JSM-7001F scanning electron microscopy (JEOL Ltd., Japan). The thermal properties of samples were determined by a diamond TG/DTA instrument (Thermo Plus-EVO, Rigaku, Japan) under a nitrogen atmosphere at a heating rate of $10{ }^{\circ} \mathrm{C} \mathrm{min}{ }^{-1}$ from $25-800{ }^{\circ} \mathrm{C}^{25}$ An UV-Vis spectrophotometer (UV-2450, Shimadzu, Japan) was employed to collect UV-Vis adsorption spectra. The sonication of samples was performed with a Branson digital $450 \mathrm{~W}$ Sonifier in experiment. The water contact angles of solid particles was measured with an optical contact angle measuring device (KSV CM200) according to the literature. ${ }^{26} \mathrm{~A}$ specific surface and pore size analysis instrument (BET/BJH method, 3H-2000PS1, Beishide Instrument Technology Co., Ltd., China) was used for the determination of nitrogen adsorption-desorption isotherms of the samples. The BET model was used to calculate the specific surface area, and $\mathrm{BJH}$ method (nitrogen desorption isotherms) was used to analyze the pore size distribution.

\subsection{Preparation of SP-MIMs and SP-MIMs}

The SP-MIMs were synthesized by GLS-stabilized Pickering emulsion polymerization strategy as follows: First, $0.1 \mathrm{mmol}$ of SP and $0.6 \mathrm{mmol}$ of MAA were dissolved in $2.0 \mathrm{~mL}$ of toluene. The mixture was kept still in dark place for $4 \mathrm{~h}$ to produce the preassembly solution. Secondly, $0.16 \mathrm{~g}$ of GLS particles were dispersed in $5.0 \mathrm{~mL}$ of doubly distilled water, and then the obtained dispersion was sonicated using a Branson $450 \mathrm{~W}$ sonifier at $50 \%$ amplitude for $20 \mathrm{~min}$ in $2.0 \mathrm{~s}$ intervals with a $3.0 \mathrm{~s}$ rest period to produce a dark brown suspension of GLS, which was used as the water phase. During the ultrasonic process, the dispersion was cooled by an ice bath in order to prevent an increase in the dispersion temperature. In order to prepare the oil phase, $2.0 \mathrm{mmol}$ of EGDMA and $15 \mathrm{mg}$ AIBN were added to the preassembly solution. The water phase was mixed with oil phase and then the mixture was violently shook by hand, a stable Pickering emulsion was prepared. Finally, the as-prepared Pickering emulsion was purged oxygen by nitrogen gas for $10 \mathrm{~min}$. Afterwards, the tiny bottles of emulsion was sealed up and then the temperature was increased to $65^{\circ} \mathrm{C}$ and kept for $24 \mathrm{~h}$. After separated by centrifuge, the doubly distilled water was added to the beaker containing the obtained products. The white solid particles were precipitate in the bottom of the beaker. After the upper dark brown mixture was poured, the white solids were washed with ethanol and then were dried at $50{ }^{\circ} \mathrm{C}$ in a vacuum drying oven. In order to remove the template molecules, the obtained powders were washed repeatedly with the solution of methanol and acetic acid $(9: 1 \mathrm{v} / \mathrm{v})$ in a Soxhlet extractor until no SP was detected in the eluent. The SP-MIMs were dried at $50{ }^{\circ} \mathrm{C}$ under vacuum. For the control 
experiments, SP-based non-imprinted microspheres (SP-NIMs) were synthesized without the addition of template molecules (SP).

\subsection{Static adsorption experiments}

Static adsorption experiments included adsorption isotherm and adsorption kinetics. For the equilibrium experiments, $10.0 \mathrm{mg}$ of SP-MIMs or SP-NIMs was respectively suspended in the solution $(10 \mathrm{~mL})$ of methanol and doubly distilled water $(1: 4, \mathrm{v} / \mathrm{v})$. And the initial concentration of SP was ranged from 10 to $200 \mathrm{mg} \mathrm{L}^{-1}$. The suspensions were placed in thermostatically controlled water at $298 \mathrm{~K}$ and $308 \mathrm{~K}$, respectively. After adsorption for $12 \mathrm{~h}$, the residual amount of SP in the solution was measured by an UV-Vis spectrophotometry at $232 \mathrm{~nm}$. The contact time had influence on adsorption of sorbents for SP, which was explored by adsorption kinetics. $10 \mathrm{mg}$ of SP-MIMs or SP-NIMs was respectively dispersed in $10 \mathrm{~mL}$ of solution and the concentration of SP was $100 \mathrm{mg} \mathrm{L^{-1 }}$. The solution was incubated at $298 \mathrm{~K}$. The adsorbent was removed from the solution at different time intervals. The residual concentrations of SP was detected by an UV-Vis spectrophotometer. The adsorption capacity $\left(Q_{t}, \mathrm{mg} \mathrm{g}^{-1}\right)$ can be calculated according to the difference of SP concentration in aqueous before and after adsorption, which can be expressed as follows: ${ }^{27}$

$$
Q_{\mathrm{t}}=\frac{\left(C_{0}-C_{\mathrm{t}}\right) V}{m}
$$

where $C_{0}\left(\mathrm{mg} \mathrm{L}^{-1}\right)$ was defined as the initial concentration of SP. $C_{t}\left(\mathrm{mg} \mathrm{L}^{-1}\right)$ was defined as the residual concentration of SP at time $t . V(\mathrm{~mL})$ represented the volume of adsorption solution and $m(\mathrm{~g})$ was the adsorbent mass. If $C_{t}$ and $Q_{t}$ were respectively replaced by $C_{\mathrm{e}}$ and $Q_{\mathrm{e}}, Q_{\mathrm{e}}\left(\mathrm{mg} \mathrm{g}^{-1}\right)$ can be calculated according to eqn (1). $Q_{\mathrm{e}}\left(\mathrm{mg} \mathrm{g}^{-1}\right)$ was the amounts of adsorbate on the sorbent at equilibrium. $C_{\mathrm{e}}\left(\mathrm{mg} \mathrm{L}^{-1}\right)$ was the equilibrium concentration of SP.

\subsection{Selective recognition experiments}

In order to investigate the selective recognition ability of SPMIMs for template molecules SP, $10 \mathrm{mg}$ of SP-MIMs or SPNIMs was separately added to $10 \mathrm{~mL}$ of solutions, and each solution contained $100 \mathrm{mg} \mathrm{L}^{-1} \mathrm{SP}$, EM, AZM or CHL, respectively. After the binding experiments were carried out at $298 \mathrm{~K}$ in water for $12 \mathrm{~h}$, the remaining amount of SP and CHL were detected at $232 \mathrm{~nm}$ and $278 \mathrm{~nm}$ by an UV-Vis spectrophotometer, respectively. The remaining amount of EM and AZM can not be measured by UV-Vis spectrophotometer. However, the mixture of a certain amount of concentrated phosphoric acid and EM or AZM solution was heated in a boiling water bath for $10 \mathrm{~min}$, the achieved solution will have a stable UV absorption peak at $482 \mathrm{~nm} \cdot .^{28}$ So the remaining amount of EM and AZM can be detected at $482 \mathrm{~nm}$ by UV-Vis spectrophotometer after treatment with concentrated phosphoric acid.

\subsection{Regeneration of adsorbent}

In order to study the regeneration and recovery of SP-MIMs, $10 \mathrm{mg}$ of the SP-MIMs was incubated in $100 \mathrm{mg} \mathrm{L}^{-1}$ of SP solution $(10 \mathrm{~mL})$ at $298 \mathrm{~K}$ for $12 \mathrm{~h}$. The SP-MIMs with adsorbed SP was washed with methanol/acetic acid $(9: 1, \mathrm{v} / \mathrm{v})$ to remove the template SP, and then dried overnight at $50{ }^{\circ} \mathrm{C}$. To investigate the regeneration, the achieved products were reused and dispersed in $10 \mathrm{~mL}$ solutions of SP with initial concentrations of $100 \mathrm{mg} \mathrm{L}^{-1}$ in new adsorption experiments. And the adsorption efficiency of SP by the regenerated materials after several adsorption-desorption cycles was investigated.

\section{Results and discussion}

\subsection{Preparation of pickering emulsion and SP-MIMs}

The schematic illustration of synthesis approach of SP-MIMs by Pickering emulsion polymerization was presented in Scheme 1. The water phase was mixed with oil phase to form Pickering emulsion. Fig. S1A $\dagger$ showed the photographs of an oil-phase dispersion and a water-phase dispersion. The dark brown aqueous dispersion of GLS was used as water phase, and the oil phase was composed of template molecules (SP), functional monomer (MAA), crosslinking agent (EGDMA) and initiator (AIBN) in toluene, which was transparent liquid. In the imprinting process, toluene was not only used as excellent solvent for monomer and template, but also a good pore formation agent, which can promote the formation of a welldeveloped pore structure in the polymer matrix. And the pore structure was benefit for the increasement of specific surface area and the binding capacity of the sorbents. ${ }^{29}$ Photographs of a Pickering emulsion before and after sharp shaking were showed in Fig. S1B and S1C. $\dagger$ From Fig. S1B, $\dagger$ the dark brown water-phase dispersion was located at the bottom of the vial and the transparent oil phase dispersion was situated at the top of the water phase. It can be seen in Fig. $\mathrm{S} 1 \mathrm{C} \dagger$ that the GLS particles-stabilized oil-in-water Pickering emulsion formed after the two-phase was mixed and shook. GLS particles were employed as the stabilizers in emulsifying process. And GLS particles moved from water phase to the oil-water interface and prevented the oil droplets merging with each other. The micrograph of Pickering emulsion droplets stabilized by GLS particles was shown in Fig. S1D, $\dagger$ which displayed the arrangement of GLS particles adsorbed to oil and water interfaces. Fig. S1D $\dagger$ showed that individual GLS particle was clearly visible on the emulsion interface. However, the GLS particles did not cover completely the surface of emulsion droplets, which was consistent with the results of research literature. ${ }^{30}$ Due to the emulsification, the o/w emulsion droplets appeared to be roughly homogeneous and uniform.

It was very vital to select suitable stabilizer for the preparation of Pickering emulsion. And the wettability of stabilizer was an important parameter in determining the formation and type of emulsion. The wettability can be expressed in term of contact angle $\theta$ of stabilizer. The solid particles $\left(\theta>90^{\circ}\right)$ was usually termed hydrophobic and can be used to stabilize water-oil emulsions, on the contrary, the particles $\left(\theta<90^{\circ}\right)$ was usually hydrophilic and suitable to stabilize oil-water emulsion. The particles with $\theta$ slightly below or beyond $90^{\circ}$ were optimal. ${ }^{31}$ The contact angle $\theta$ of GLS had been measured and displayed in Fig. S2A. $\dagger$ The contact angle $\theta$ of GLS was $31.45^{\circ}$, which indicate 


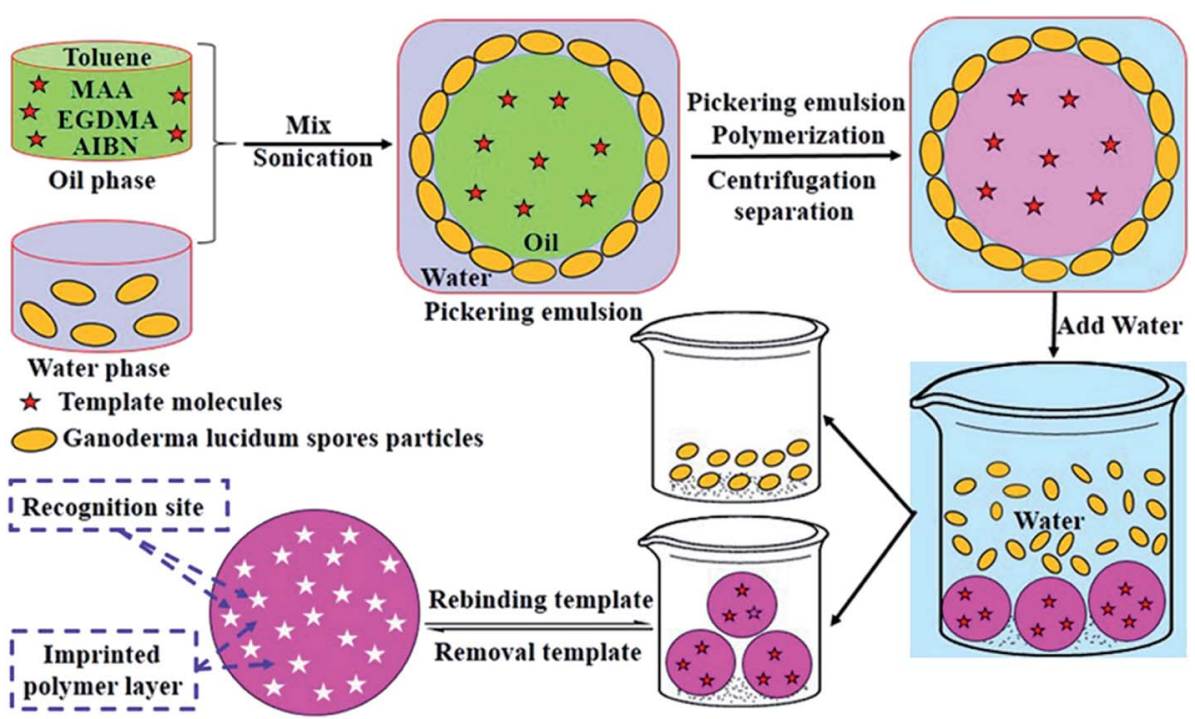

imprinted microspheres

Scheme 1 Sketches of fabrication approach of SP-MIMs by Pickering emulsion polymerization.

that the GLS particles were extremely hydrophilic. According to the value of $\theta$, GLS particles seemed to be unsuitable for the use as stabilizer. However, it was satisfying that the GLS particles could move from water phase to the oil-water interface and stabilize the emulsion. It was reported by Wang et al. that the IR spectra of GLS at different areas were similar and GLS contained the ingredient of polysaccharide, sterols, proteins, fatty acids, etc. ${ }^{22}$ As a natural biological material, GLS contained various organic ingredient, which was different from those inorganic materials. When GLS were used to stabilize Pickering emulsion, the particularity of GLS, including the organic ingredient and special structure, maybe promote the movement of GLS particles from water phase to oil phase. And the contact angle $\theta$ of GLS was not the determinants of the formation of Pickering emulsion. Thus a Pickering emulsion was formed with the action of GLS particles.

In the imprinting system, SP, MAA, EGDMA and AIBN were chosen as the template molecules, functional monomer, crosslinking agent and initiator, respectively. The selfassembled system was firstly formed between MAA and template SP because of the hydrogen bonding interaction. Then, free radical polymerization between functional monomers (MAA) and crosslinker (EGDMA) was initiated by AIBN under heating, and then polymer networks gradually formed. After the polymerization reaction ended, the doubly distilled water was added to the as-prepared solid in a beaker. Surprisingly, the white solid particles precipitated out in the bottom of the beaker. Fig. $\mathrm{S} 2 \mathrm{~B} \uparrow$ showed the photograph of the desquamating of GLS particles and the sedimentation of polymer microspheres. Obviously, GLS particles had dropped off from the surface of the imprinted polymer microspheres, which might be ascribed to the hydrophilia. And then the deciduous GLS particles were suspended in the water. Although, GLS particles could move from water to the water-oil interface in the formation process of the emulsion, GLS particles were so hydrophilic that they could not be wetted well by oil phase. The result was that GLS particles were merely attached to the surface of imprinted microspheres and easily came off the imprinted microspheres. The GLS particles and SP-MIMs were easily separated because the GLS particles were suspended in water and polymer microspheres precipitated to the bottom of the beaker. In order to confirm the complete separation of GLS and polymer microspheres, the surface condition of the polymer microspheres sitting in the bottom of the beaker was investigated using the microscope, as shown in Fig. S2C. $\dagger$ It could be seen that there were still some GLS particles on the surface of polymer microspheres, indicating that GLS particles and polymer microspheres were not completely separated. Meanwhile, the full shape of GLS particles which attached to imprinted polymeric microspheres also showed that GLS were stable in the organic solvents as well as acids in heating condition in this paper. After water was added to the preceding products and the container was shaken again, under the microscope, the bare polymer particles were obtained eventually, as shown in Fig. S2D. $\uparrow$ After the template molecules in the bare polymer microspheres were eluted by organic solvent, binding sites were produced in the polymer networks which were structurally complementary to the template molecules, thus SP-MIMs were successfully prepared.

\subsection{The influence of stable particles on the surface of imprinting microsphere}

So far, there had been many researches on the MIPs which were prepared by Pickering emulsion polymerization..$^{26,32,33}$ These MIPs were usually covered with a layer of solid particles which were used as stabilizers in emulsion. Here, the important function of solid particles was to stabilize the Pickering emulsion and facilitate the formation of spherical MIPs. In the 
adsorption experiments, solid particles covering of the surface of MIPs had no selective recognition for template molecules. On the contrary, the solid particles may have a negative effect on the adsorption performance and selective recognition ability of MIPs. Scheme 2 showed the extraction and rebinding process of template molecules on MIPs with and without stabilizers. For MIPs with stabilizers, the solid particles may influence the extracting efficiency and rebinding ability of MIPs, because the layer of solid particles had the potential transmission resistance. However, for MIPs without stabilizers, because there was no a layer of solid particles and no potential transmission resistance, the template molecules can be easily and smoothly removed by eluting solvent or rebound by recognition sites on MIPs. So the selective adsorption capacity of the MIPs without stabilizers may be superior to the MIPs with stabilizers. Thus, it was very interesting and important to achieve the MIPs without stabilizers by very simple means. Because there was no the blocking and disturbance of GLS particles on the surface of SPMIMs, the elution and rebinding efficiency of template molecules might be dramatically improved.

\subsection{The effect of GLS amount on the pickering emulsion}

The effect of GLS amount on the stability of Pickering emulsion was investigated. In Fig. S3A, $\uparrow$ the volume of toluene and water were $3.0 \mathrm{~mL}$ and $7.0 \mathrm{~mL}$ respectively, the amount GLS was $0.10 \mathrm{~g}$. However, the oil phase was rapidly separated from the water phase and the GLS particles were fully dispersible in water, so the Pickering emulsion was enormously unstable under these conditions. The volume of oil and water phase were remained unchanged, but the amount of GLS particles was increased to $0.16 \mathrm{~g}$, the stability of emulsion was improved in a certain extent, but the emulsion breaking and delamination could be seen in Fig. S3B. $\dagger$ When the dosage of toluene and water were $2.0 \mathrm{~mL}$ and $5.0 \mathrm{~mL}$ respectively, and the material mass of GLS was still $0.16 \mathrm{~g}$, the stable Pickering emulsion was prepared on the same operation. Thus, it was found that increasing the amount of GLS would contribute to the improvement of Pickering emulsion stability (Fig. S3C $\dagger$ ).

\subsection{Characterization of imprinted microspheres}

3.4.1. Morphology characterization of polymers. In this experiment, the GLS were produced by the Ganoderma lucidum in northeast China, and Fig. 1A revealed the photograph of the GL. SEM images of GLS were presented in Fig. 1B and C. The spores were oval shape, which were about 4.9 and $8.3 \mu \mathrm{m}$ in width and length, respectively. Moreover, the top of GLS shows truncation or blunt conoid, and there were several holes on the surface of the spores. ${ }^{34}$ Fu et al. ${ }^{35}$ also studied the morphology of broken spores, and found that the GLS were of hollow structure. It was this special structure that enabled the GLS particles to suspend in the water after dropping out from the surface of microspheres. The SEM images of SP-MIMs were shown in Fig. 1D and E. SP-MIMs presented regular sphericity and their size was about 60-70 $\mu \mathrm{m}$. It seemed to be rough. And there were sporadic spores attaching to the surface of only a few microspheres, which not only provided evidence that GLS particles were located at the surface of imprinted polymer microspheres after emulsion polymerization, but also indicated that spores and microspheres were separated completely. Moreover, it was obvious that the shedding of GLS made no imprint on the surface of microspheres. From this, we inferred further that the GLS particles only accumulated mainly at oil-water interface in Pickering emulsion, instead of being immersed into oil phase, which result in the easy separation between GLS and polymer microspheres. A more detailed observation by SEM (Fig. 1F) revealed that the surface of polymers were uncovered due to the removal of GLS. The rough and loose surface of polymers were not only helpful for enhancing the special area of SP-MIMs, but also in favor of removing and rebinding of template molecules.

3.4.2. FT-IR spectra analysis. FT-IR spectra of GLS, SPMIMs and SP-NIMs were measured and showed in Fig. 2. According to the spectrum of GLS in Fig. 2A, the broad

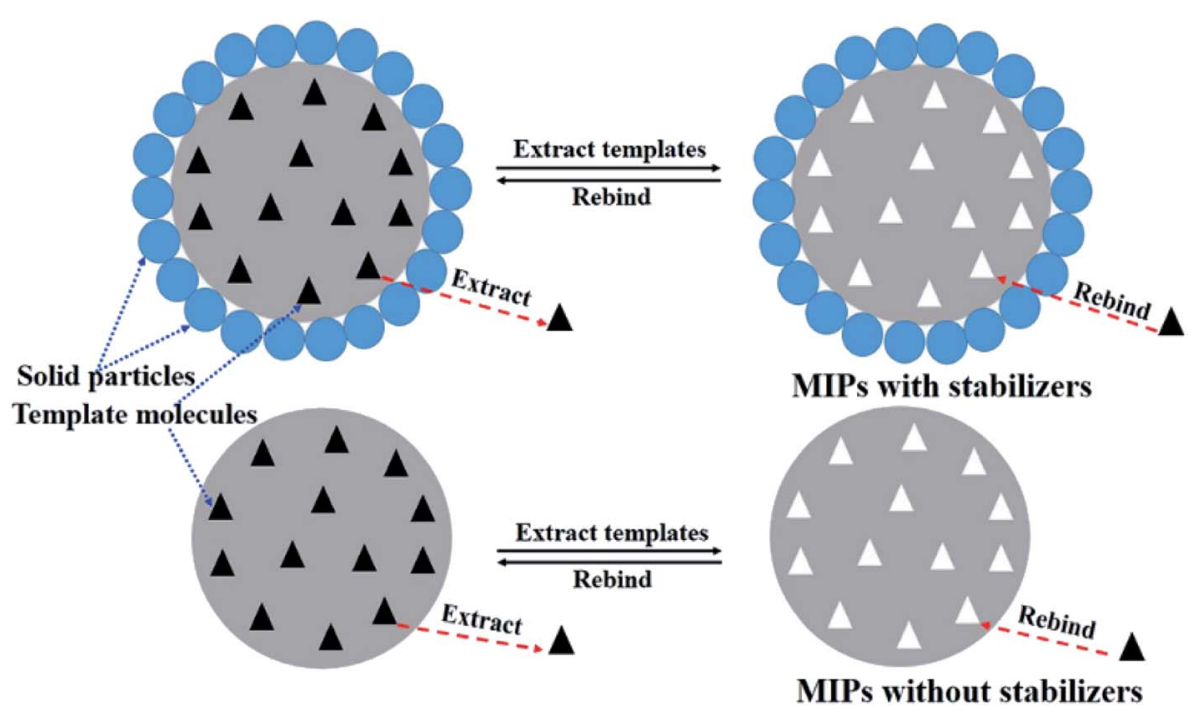

Scheme 2 Sketches of the impacts of stabilizer particles on MIPs prepared by Pickering emulsion. 
absorption band at $3434 \mathrm{~cm}^{-1}$ was attributed to the vibration of $\mathrm{N}-\mathrm{H}$ group and the absorption bands at 2924 and $2853 \mathrm{~cm}^{-1}$ were assigned mainly to asymmetric and symmetric stretching vibrations of $-\mathrm{CH}_{3}$ and $-\mathrm{CH}_{2}-$. Besides, a sharp peak was presented at $1746 \mathrm{~cm}^{-1}$ which could be ascribed to the $\mathrm{C}=\mathrm{O}$ stretching in ester. ${ }^{24}$ The bands at $1638 \mathrm{~cm}^{-1}$ and $1151 \mathrm{~cm}^{-1}$ were respectively assigned to the absorption of $-\mathrm{CO}-\mathrm{NH}_{2}$ and stretching of $\mathrm{C}-\mathrm{O}$ in proteins and carbohydrates, and the wide band at $1077 \mathrm{~cm}^{-1}$ was a typical characteristic of sugars. ${ }^{22}$ Thus it could be seen that GLS contained various organic functional groups. There were several adsorption peaks in the spectra of SP-MIMs at $3461 \mathrm{~cm}^{-1}, 2954 \mathrm{~cm}^{-1}, 1729 \mathrm{~cm}^{-1}, 1454 \mathrm{~cm}^{-1}$,
$1386 \mathrm{~cm}^{-1}, 1262 \mathrm{~cm}^{-1}$ and $1155 \mathrm{~cm}^{-1}$, respectively, as shown in Fig. 2B. Among them, the broad absorption band at $3461 \mathrm{~cm}^{-1}$ was attributed to the vibration of $\mathrm{O}-\mathrm{H}$ group. The broad absorption band at $2954 \mathrm{~cm}^{-1}$ was assigned to the $\mathrm{C}-\mathrm{H}$ asymmetry stretching vibrations of saturated hydrocarbons (both $\mathrm{CH}_{3}$ and $\mathrm{CH}_{2}$ groups). And the strong absorption at $1729 \mathrm{~cm}^{-1}$ was referred to the $\mathrm{C}=\mathrm{O}$ stretching vibrations of EGDMA and MAA. The distinct adsorption peak at $1454 \mathrm{~cm}^{-1}$ can be assigned to asymmetric stretching in-plane flexural vibration of $\mathrm{C}-\mathrm{H}\left(-\mathrm{CH}_{3}\right.$ or $\left.-\mathrm{CH}_{2}-\right)$, and the peak at $1386 \mathrm{~cm}^{-1}$ was assigned to symmetric stretching in-plane flexural vibration of $\mathrm{C}-\mathrm{H}$ $\left(-\mathrm{CH}_{3}\right)$ of EGDMA and MAA. The FT-IR peaks at $1262 \mathrm{~cm}^{-1}$ and
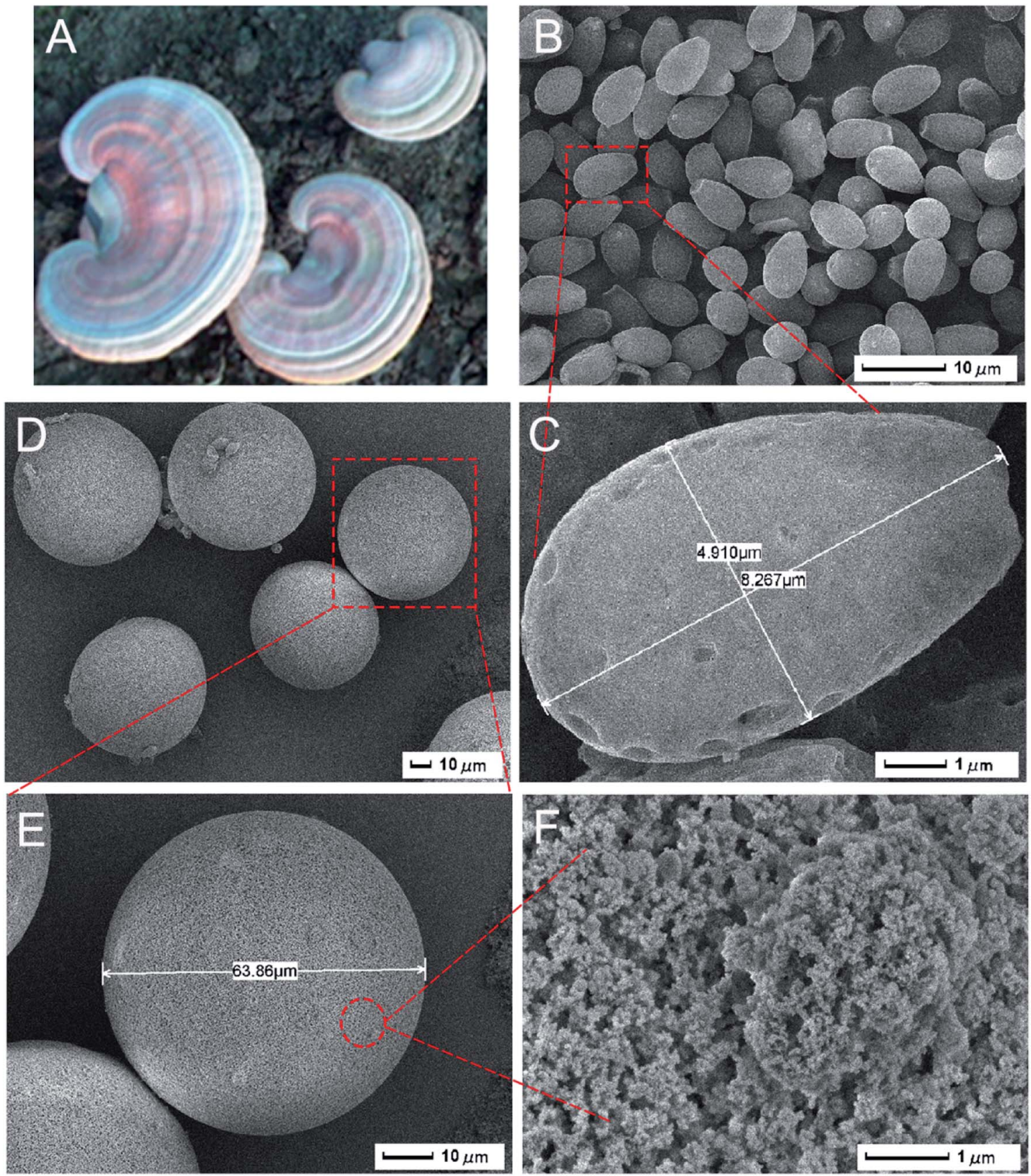

Fig. 1 The picture of GL (A) and SEM images of GLS (B and C), SP-MIMs (D and E) and the magnification image of its surface (F). 
$1155 \mathrm{~cm}^{-1}$ were ascribe to the $\mathrm{C}-\mathrm{C}(=\mathrm{O})-\mathrm{O}$ absorption peaks of the saturated ester which came from EGDMA. Furthermore, the adsorption peak at $1637 \mathrm{~cm}^{-1}$ in Fig. 2B could be imputed to $\mathrm{C}=\mathrm{C}$ vibration of EGDMA, suggesting that the bonds of the EGDMA molecules were not $100 \%$ cross-linked in the SPMIMs. ${ }^{36}$ Most importantly, there was no adsorption peak at $1077 \mathrm{~cm}^{-1}$ in Fig. 2B, which was the characteristic adsorption peak of sugars in GLS, indicating that the absence of GLS particles on the surface of SP-MIMs. Furthermore, it was observed in Fig. 2C that the FT-IR spectrum of SP-NIMs was nearly identical to that of SP-MIMs, which indicated that the template molecules had been removed completely.

3.4.3. Nitrogen adsorption-desorption analysis. The surface properties of adsorbing materials had a big impact on their adsorptive properties in the adsorption process. So the porosity of GLS, SP-MIMs and SP-NIMs was also studied by nitrogen adsorption-desorption measurements. And the test data of BET specific surface area $\left(S_{\mathrm{BET}}\right)$, cumulative pore volume $\left(V_{\mathrm{c}}\right)$ and average pore diameter $\left(D_{\mathrm{p}}\right)$ of the studied materials were listed in Table S1. $\dagger$ The $S_{\mathrm{BET}}, V_{\mathrm{c}}$ and $D_{\mathrm{p}}$ for the GLS were $2.77 \mathrm{~m}^{2} \mathrm{~g}^{-1}, 0.0461 \mathrm{~mL} \mathrm{~g}^{-1}$ and $14.11 \mathrm{~nm}$, respectively. Comparing the imprinted and the non-imprinted polymers, it was obvious that the $S_{\mathrm{BET}}$ and $V_{\mathrm{c}}$ of SP-MIMs $\left(374.78 \mathrm{~m}^{2} \mathrm{~g}^{-1}\right.$ and $0.8027 \mathrm{~mL} \mathrm{~g}^{-1}$ ) were slightly higher than those of SP-NIMs $\left(309.22 \mathrm{~m}^{2} \mathrm{~g}^{-1}\right.$ and $0.7225 \mathrm{~mL} \mathrm{~g}^{-1}$ ), respectively. The $D_{\mathrm{p}}$ of SPMIMs $(11.25 \mathrm{~nm})$ were also slightly larger than the $D_{\mathrm{p}}$ of SPNIMs $(10.12 \mathrm{~nm})$. These results indicated that the template molecules had significant impact on the structure of imprinted and non-imprinted polymers. In other words, these results directly implied that SP-MIMs had higher number of selective binding sites as a result of the imprinting effect. ${ }^{37}$

3.4.4. Thermogravimetric analysis. Thermogravimetric analysis (TGA) was the most common method to determine the thermal stability of materials. The TGA curves of SP-MIMs (A) and SP-NIMs (B) were given in Fig. 3. As shown in Fig. 3, when the temperature was less than $150{ }^{\circ} \mathrm{C}$, there was a small weight loss of about $7.71 \%$ (SP-MIMs) and $6.69 \%$ (SP-NIMs), respectively, which were attributed to the loss of residual water. The

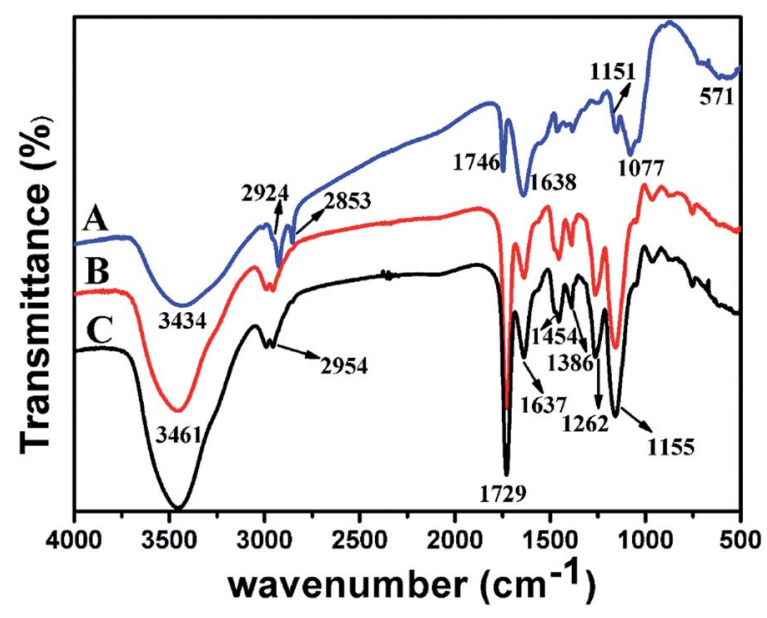

Fig. 2 FT-IR spectra of GLS (A), SP-MIMS (B) and SP-NIMS (C). weight loss of SP-MIMs or SP-NIMs was very small between $150{ }^{\circ} \mathrm{C}$ and $200{ }^{\circ} \mathrm{C}$. Then there was a sharp reduction in the weight of SP-MIMs and SP-NIMs after the temperature reached to $250{ }^{\circ} \mathrm{C}$. With the temperature increased to $450{ }^{\circ} \mathrm{C}$, the significant weight losses of SP-MIMs (74.37\%) and SP-NIMs (73.46\%) could be observed, which could be due to the loss of the polymer in SP-MIMs and SP-NIMs. Above $450{ }^{\circ} \mathrm{C}$, the weight of the each specimen was constant and the residual mass for SPMIMs and SP-NIMs might be ascribed to the thermal resistance of residual carbon by calcining polymers. ${ }^{6}$ Moreover, the TGA curves of SP-MIMs and SP-NIMs had followed the same trend, indicating that the imprinted and non-imprinted polymers possessed similar morphological structure and size distributions. ${ }^{6}$ The result of TGA curve showed that the as-prepared imprinted sorbents possessed thermal stability (below $250{ }^{\circ} \mathrm{C}$ ).

\subsection{Adsorption isotherm}

In order to evaluate the adsorption capacity of the as-prepared imprinted and non-imprinted polymers for the template SP, adsorption capacity of SP-MIMs and SP-NIMs at different initial concentration of SP and temperature was measured, and the results were shown in Fig. 4. As can be seen from the chart, the adsorption capacity of SP-MIMs (Fig. 4A) had the similar tendency of changes with that of SP-NIMs (Fig. 4B). The amount of SP adsorbed on the two sorbents was also found to increase primitively along with increasing the initial concentration of SP, and then gradually attain saturation. Fig. 4 also showed that the adsorption amounts of SP-MIMs and SP-NIMs for SP at $308 \mathrm{~K}$ were greater than the amounts at $298 \mathrm{~K}$, which indicated that the temperature had an impact on the SP adsorption and the adsorbed amount of SP increased with temperature. So the increase in temperature would be benefit for the adsorption. In addition, it was seen from Fig. 4 that the amounts of SP adsorbed on the SP-MIMs were higher than the SP-NIMs under the same conditions. Because the only difference between SPMIMs and SP-NIMs was whether or not adding the template molecule in the process of preparation, the difference of adsorption quantity between the two kinds of adsorbents was

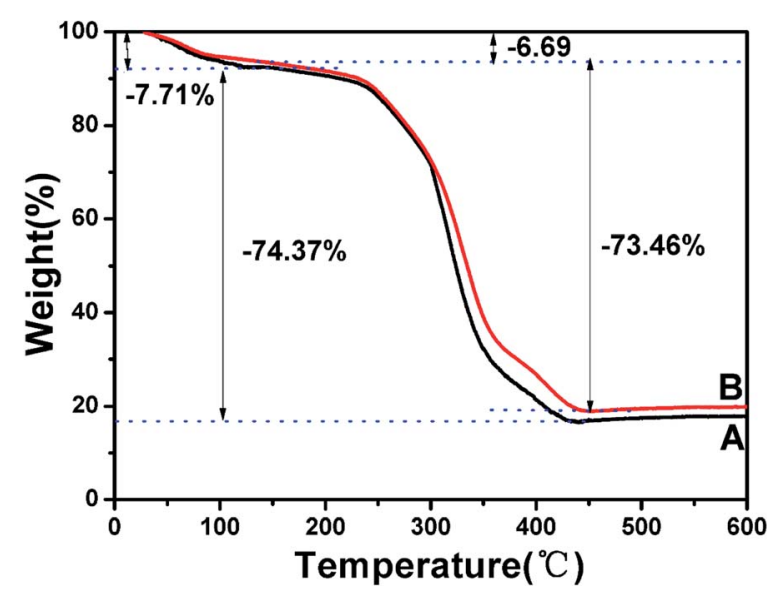

Fig. 3 TGA curves of SP-MIMs (A) and SP-NIMs (B). 

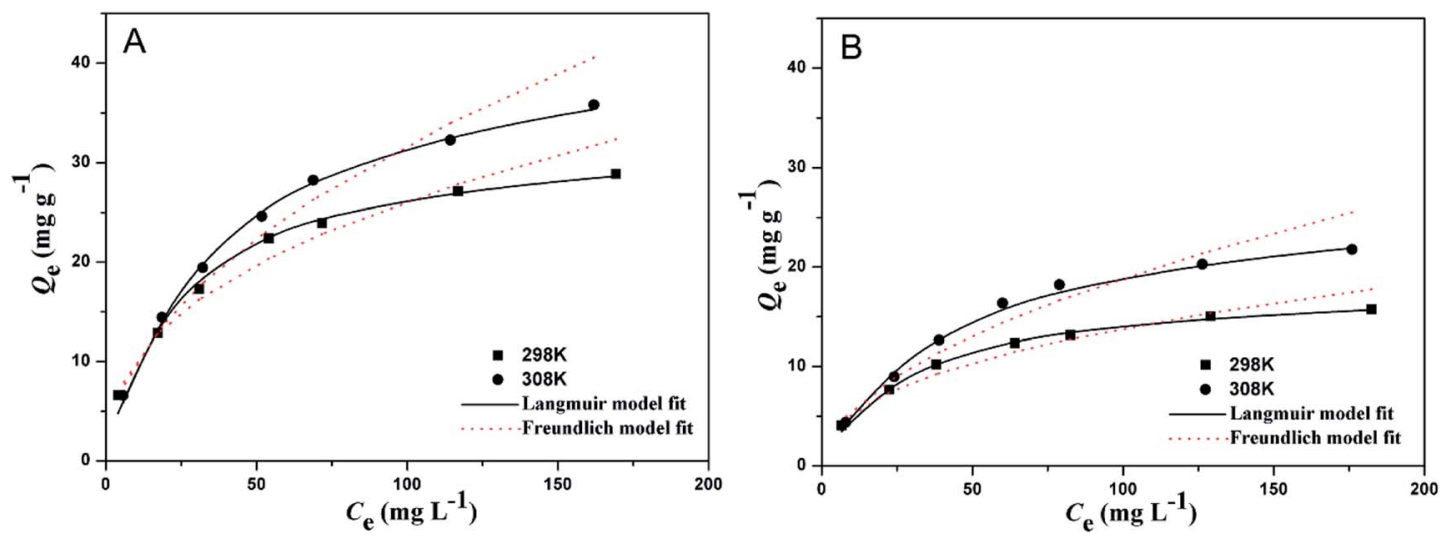

Fig. 4 The adsorption isotherms of SP onto SP-MIMs (A) and SP-NIMs (B) under $298 \mathrm{~K}$ and $308 \mathrm{~K}$.

mainly caused by a great many specific binding sites for SP on the SP-MIMs. Besides, to study further the binding properties of adsorbents, Langmuir isotherm model and Freundlich model were used to fit the equilibrium data of adsorption experiments, ${ }^{38,39}$ and the results were displayed in Fig. 4. The nonlinear expression of Langmuir and Freundlich isotherm equations were expressed by eqn (2) and (3), respectively.

$$
Q_{\mathrm{e}}=\frac{K_{\mathrm{L}} Q_{\mathrm{m}} C_{\mathrm{e}}}{1+K_{\mathrm{L}} C_{\mathrm{e}}}
$$

where $Q_{\mathrm{e}}\left(\mathrm{mg} \mathrm{g}^{-1}\right)$ is the amount of SP adsorbed per unit adsorbent mass at equilibrium, $Q_{\mathrm{m}}\left(\mathrm{mg} \mathrm{g}^{-1}\right)$ is a constant related to the maximum sorption capacity, $C_{\mathrm{e}}\left(\mathrm{mg} \mathrm{L}^{-1}\right)$ is the concentration of SP in solution at equilibrium, and $K_{\mathrm{L}}\left(\mathrm{L} \mathrm{mg}^{-1}\right)$ is the Langmuir equilibrium constant.

$$
Q_{\mathrm{e}}=K_{\mathrm{F}} C_{\mathrm{e}}^{1 / n}
$$

where $K_{\mathrm{F}}\left(\mathrm{L}^{1 / n} \mathrm{mg}^{(1-1 / n)} \mathrm{g}^{-1}\right)$ is the constant of the Freundlich isotherm, $n$ also represents Freundlich constant, $1 / n$ is the Freundlich exponent and also a measure of exchange intensity or surface heterogeneity, and a value of $1 / n$ smaller than 1.0 can reflect favorable removal conditions. ${ }^{38,40}$ In order to evaluate the favorability of a sorption system, the affinity constant $R_{\mathrm{L}}$ is introduced to express the Langmuir equation in terms of a dimensionless separation factor, which can be defined as follows: ${ }^{6}$

$$
R_{\mathrm{L}}=\frac{1}{1+C_{\mathrm{m}} L_{\mathrm{L}}}
$$

where $C_{\mathrm{m}}\left(\mathrm{mg} \mathrm{L}^{-1}\right)$ is the maximal initial concentration of SP. The values of $R_{\mathrm{L}}$ represent the favorability and the capacity of adsorption system. If $0<R_{\mathrm{L}}<1.0$, the values of $R_{\mathrm{L}}$ will indicate a good adsorption.

Adsorption equilibrium parameters were calculated according to Langmuir and Freundlich models, and the results were shown in Table 1. Among them, $R^{2}$ was the correlation coefficient of the two models, and the value of $R^{2}$ can be used to estimate the validity of each model. Obviously, when the temperature was $298 \mathrm{~K}$ and $308 \mathrm{~K}$, the value of $R^{2}$ for the Langmuir isotherm model were greater than those of Freundlich model, implying that the Langmuir isotherm model could better describe SP adsorption onto SP-MIMs and SP-NIMs than the Freundlich isotherm model. And the results also indicated that the as-prepared sorbents had uniform solid surface, and the adsorption of SP molecules on the sorbents was a regular monolayer adsorption process. ${ }^{6}$ Moreover, according to Table 1 , the value of $R_{\mathrm{L}}$ were between 0 and 1 , the value of $1 / n$ were less than 1 , which suggested that the experiment conditions were benefit for adsorption of SP. However, the value of $R_{\mathrm{L}}$ for SPMIMs were slightly less than those of SP-NIMs at the same temperature, indicating the removal condition of SP-MIMs was more favorable.

\subsection{Adsorption kinetic}

In order to evaluate the adsorption efficiency of as-prepared adsorbent, adsorption kinetics experiments were carried out under $298 \mathrm{~K}$. The related kinetic adsorption data of SP onto SP-

\begin{tabular}{|c|c|c|c|c|c|c|c|c|}
\hline Sorbents & $T(\mathrm{~K})$ & \multicolumn{4}{|c|}{ Langmuir isotherm equation } & \multicolumn{3}{|c|}{ Freundlich isotherm equation } \\
\hline & 308 & 43.48 & 0.0269 & 0.1565 & 0.9972 & 3.071 & 0.5073 & 0.9734 \\
\hline \multirow[t]{2}{*}{ SP-NIMs } & 298 & 18.08 & 0.0357 & 0.1229 & 0.9980 & 2.035 & 0.4160 & 0.9692 \\
\hline & 308 & 27.40 & 0.0228 & 0.1799 & 0.9968 & 1.630 & 0.5321 & 0.9606 \\
\hline
\end{tabular}
MIMs and SP-NIMs were displayed in Fig. 5. Obviously, the

Table 1 The related fitting parameters of two isotherm equations for SP adsorption onto SP-MIMs and SP-NIMS 


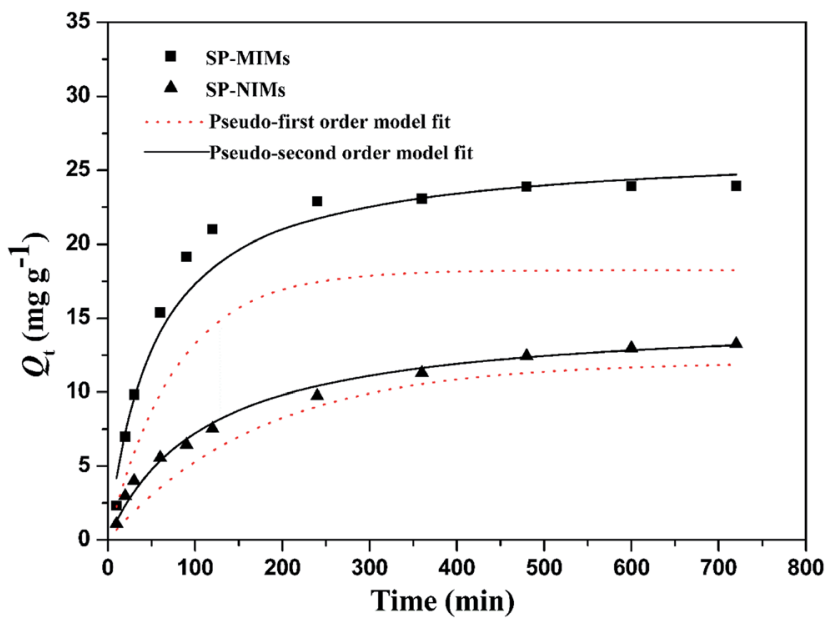

Fig. 5 Kinetic for the adsorption of SP onto SP-MIMs and SP-NIMS under $298 \mathrm{~K}$.

kinetic adsorption process of SP-MIMs and SP-NIMs for SP followed the same varying tendency. The adsorption capacity of SP-MIMs and SP-NIMs for SP increased rapidly in the first $100 \mathrm{~min}$, and the reason was that the adsorbates (SP) could be easily diffused through the porous adsorbents at a high particle initial concentration condition. ${ }^{38}$ And then the adsorption speed of SP-MIMs and SP-NIMs decreased gradually, the corresponding adsorbed amount slowly increased until it reached an equilibrium. However, Fig. 5 also showed that SP-MIMs exhibited a much more adsorption equilibrium capacities than SP-NIMs, and all of the adsorption capacity data of SPMIMs for SP were obviously much higher than those of SPNIMs in the same conditions. It could be seen in Fig. 5 that the adsorption rate of SP-MIMs was faster than that of SP-NIMs. These results suggested that a large number of special imprinted binding sites had been formed on SP-MIMs and these imprinted sites possessed high selectivity and affinity to SP. In addition, for further research on the adsorption mechanism, pseudo-first-order and pseudo-second-order kinetic equations were chosen to analyze the adsorption kinetics data of SP-MIMs and SP-NIMs for SP. And the pseudo-first-order and pseudosecond-order kinetic equations could be expressed respectively by the eqn (5) and (6): ${ }^{41}$

$$
\begin{gathered}
Q_{t}=Q_{\mathrm{e}}-Q_{\mathrm{e}^{-}}{ }^{-k_{1} t} \\
Q_{t}=\frac{K_{2} Q_{\mathrm{e}}{ }^{2} t}{1+k_{2} Q_{\mathrm{e}} t}
\end{gathered}
$$

where $Q_{t}\left(\mathrm{mg} \mathrm{g}^{-1}\right)$ is the amount of SP adsorbed at time $t$ (min) and $Q_{\mathrm{e}}\left(\mathrm{mg} \mathrm{g}^{-1}\right)$ is the adsorption capacity for SP at equilibrium. $k_{1}\left(\mathrm{~min}^{-1}\right)$ and $k_{2}\left(\mathrm{~g} \mathrm{mg}^{-1} \mathrm{~min}^{-1}\right)$ are respectively the rate constants of the pseudo-first and pseudo-second kinetic equations. The values of $k_{1}$ can be calculated from the plots of $\ln \left(Q_{\mathrm{e}}\right.$ $\left.-Q_{t}\right)$ versus $t$ and the values of $k_{2}$ can be computed from the plots of $t / Q_{t}$ versus $t$. According to the pseudo-second-order kinetic model, the values of initial adsorption rate $h(\mathrm{mg}$ $\mathrm{g}^{-1} \min ^{-1}$ ) and half equilibrium time $t_{1 / 2}(\mathrm{~min})$ can be calculated by eqn (7) and (8), ${ }^{42}$ respectively.

$$
\begin{aligned}
& h=K_{2} Q_{\mathrm{e}}{ }^{2} \\
& t_{1 / 2}=\frac{1}{k_{2} Q_{\mathrm{e}}}
\end{aligned}
$$

The fitting curves by the pseudo-first-order and pseudosecond-order kinetic models were shown in Fig. 5, and the fitting parameters along with the correlation coefficient $\left(R^{2}\right)$ of the two kinetic equations were summarized in Table 2. It was clearly observed from Fig. 5 that the kinetic adsorption data of SP-MIMs and SP-NIMs were fitted well with the pseudo-secondorder kinetic model. Furthermore, by comparing and analyzing the values of correlation coefficient $R^{2}$ in Table 2, it was evident that the kinetic adsorption process of both SP-MIMs and SPNIMs followed the pseudo-second-order kinetics model $\left(R^{2}>\right.$ 0.99), which agreed with the conclusion from Fig. 5. It was concluded that the adsorption between SP-MIMs or SP-NIMs and SP was a chemisorption process, which could be the ratelimiting step during the adsorption for SP. ${ }^{43}$ And the numeric comparison of $h$ and $t_{1 / 2}$ in Table 2 also suggested that SP-MIMs possessed more excellent kinetic properties than SP-NIMs, because SP-MIMs had much faster initial adsorption rate and shorter half equilibrium time. This advantage of SP-MIMs could also be ascribed to the successful formation of imprinted binding sites on SP-MIMs.

\subsection{Selectivity analysis}

Excellent selective recognition ability was an important indicator to measure the adsorption performance of MIPs for template molecules. Besides the template SP, the other three antibiotics (AZM, CHL and EM) were selected as comparison to investigate the adsorption specificity of as-prepared adsorbents. Among them, EM and AZM were respectively 14-membered and 15-membered ring macrolides antibiotics, and their structure were similar to SP. The molecular weight of CHL was relatively small and it had a different structure from SP. The adsorption

Table 2 Kinetic constants of two rate equations for SP adsorption onto SP-MIMs and SP-NIMs

Pseudo-first-order equation

$Q_{\mathrm{e}, \mathrm{cal}}\left(\mathrm{mg} \mathrm{g}^{-1}\right) \quad k_{1}\left(\mathrm{~min}^{-1}\right) \quad R^{2}$

Sorbents $Q_{\mathrm{e}, \exp }\left(\mathrm{mg} \mathrm{g}^{-1}\right)$

SP-MIMs $\quad 23.94$

SP-NIMs 13.25
0.0113

0.0058
Pseudo-second-order equation

$h$

$Q_{\mathrm{e}, \mathrm{cal}}\left(\mathrm{mg} \mathrm{g}^{-1}\right) \quad k_{2}\left(\mathrm{~g} \mathrm{mg}^{-1} \mathrm{~min}^{-1}\right) \quad\left(\mathrm{mg} \mathrm{g}^{-1} \min ^{-1}\right) \quad t_{1 / 2}(\mathrm{~min}) \quad R^{2}$

$\begin{array}{lll}0.9675 & 26.53 & 0.0007088\end{array}$

$0.9845 \quad 15.17$

0.0005998

0.4987

53.18

109.87

0.9904

0.9954 
capacities of SP-MIMs and SP-NIMs for these four targets were presented in Fig. 6. Obviously, the binding capacity of SP-MIMs for diverse target molecules was different under the same conditions. The adsorption amounts of SP-MIMs for four testing compounds from high to low in order was: SP, AZM, EM and CHL. The difference between the amounts of AZM, EM and CHL adsorbed on SP-MIMs was less, indicating that SP-MIMs possessed the specific recognition and adsorption ability for SP. Besides, as compared to SP-MIMs, the amount of SP adsorbed on SP-NIMs was not dramatically different from that of AZM, EM and CHL, suggesting that SP-NIMs had low selectivity. The four tested target compounds had different molecular structures, and many recognition sites were formed on SPMIMs, which could better match with SP in shape, size, space structures and functional groups, so SP-MIMs had the excellent selective recognition ability for SP. Furthermore, according to the literature, ${ }^{26,38}$ the selectivity of molecularly imprinting materials was also measured by the imprinting factor $\alpha$, which was calculated by eqn (9).

$$
\alpha=\frac{Q_{\mathrm{M}}}{Q_{\mathrm{N}}}
$$

$Q_{\mathrm{M}}$ and $Q_{\mathrm{N}}$ are respectively the binding capacity of SP-MIMs and SP-NIMs. By calculation, the imprinting factor $\alpha$ were respectively 2.003, 1.368, 1.298 and 1.212 for SP, AZM, CHL and EM. These results further indicated SP-MIMs were specific for SP, but non-specific for other test compounds. Moreover, in our previous work, Erythromycin-based molecularly imprinted polymers (EM-MIPs) had been prepared by the same synthetic strategy. ${ }^{44}$ The surfaces of EM-MIPs were covered with eggshell particles and the calculated value of the imprinting factor $\alpha$ was 1.471 for template molecules. These results suggested that the bare SP-MIMs ( $\alpha$ was 2.003) possessed better selective recognition ability than EM-MIPs ( $\alpha$ was 1.471). Thus, in this Pickering emulsion polymerization strategy, the absence of stabilizers particles could greatly improve the specific recognition of imprinted microspheres for template molecules, because this special structure was benefit for the exposure of recognition sites and the elimination of the rebinding barriers between the recognition sites and template molecules. In other word, the SPMIMs derived from GLS particles-stabilized Pickering emulsion

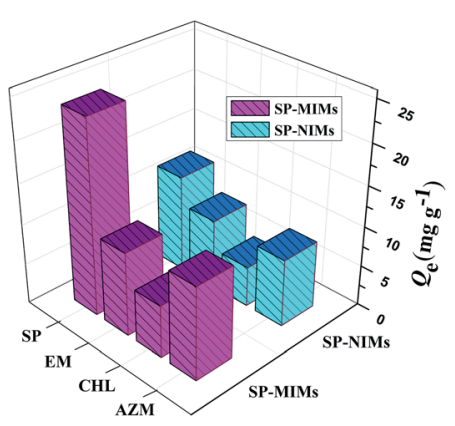

Fig. 6 Selective adsorption capacity of SP-MIMs and SP-NIMs for SP, $\mathrm{EM}, \mathrm{CHL}$ and $\mathrm{AZM}$. polymerization could effectively improve the adsorption selectivity by removing the impact of stabilized particles.

\subsection{Regeneration analysis}

The as-prepared materials will be applied effectively and widely if they can be reused many times. The sequential adsorptiondesorption cycle test were further performed in order to evaluate the regenerability of the prepared SP-MIMs. As shown in Fig. S4, $\uparrow$ the amounts of SP adsorbed on SP-MIMs diminished slightly after every adsorption-desorption cycle, and the adsorption capacity of SP-MIMs for SP after five regeneration cycles was about $89.28 \%$ of the adsorbed amount for the first use. The slight decrease of adsorbance could be ascribed as the slight reduction of binding sites. In summary, the as-prepared SP-MIMs had a satisfactory regeneration ability and also a potential application prospect in practice.

\section{Conclusion}

In conclusion, spiramycin-based molecularly imprinted microspheres (SP-MIMs) with a high density of specific recognition sites for SP were successfully prepared by Pickering emulsion polymerization. In this study, the hollow-structure and micronsize Ganoderma lucidum spores were selected as oil-in-water Pickering emulsion stabilizers for the first time. Intriguingly, there was no solid particles on the surface of SP-MIMs, which was different from the molecularly imprinted polymers synthesised by Pickering emulsion polymerization in previous studies. ${ }^{6,7,38,45}$ Moreover, the recognition adsorption property of SP-MIMs for SP in aqueous solution was estimate by adsorption isotherm, kinetic, selectivity and regeneration analysis. SPMIMs derived from Pickering emulsion possessed the following interesting features: firstly, the absence of stable particles on the surface of SP-MIMs can contribute to the extracting and rebinding of template molecules and can help mitigate the impact of stabilizer particles on the recognition sites, which can greatly enhance the selective recognition ability of SP-MIMs. Secondly, SP-MIMs, prepared by micron-size GLSstabilized emulsion polymerization, were homogeneous micrometer-sized spheres, which was helpful for their re-use for many times without large loss. Thirdly, the adsorption analysis result revealed that the Langmuir isothermal model and pseudo-second-order kinetics model were respectively suitable for fitting the adsorption equilibrium and kinetic data. Furthermore, the as-prepared SP-MIMs had good thermal stability and favorable regeneration property, and could selectively recognize and remove SP molecules in aqueous solutions. In a word, because of the high separation selectivity combined with the good regenerability, SP-MIMs based on the GLSstabilized Pickering emulsion polymerization can be a promising and reliable candidate for the recognition and adsorption of the organic pollutants.

\section{Conflicts of interest}

There are no conflicts of interest to declare. 


\section{Acknowledgements}

This study received financial support from the National Natural Science Foundation of China (No. 21676127, 21406085), Natural Science Foundation of Jilin Province (No. 20170101165JC), Science and Technology Research Project of Education Department of Jilin Province (No. 2016226).

\section{References}

1 R. Hirsch, T. Ternes, K. Haberer and K.-L. Kratz, Occurrence of antibiotics in the aquatic environment, Sci. Total Environ., 1999, 225, 109.

2 S. George, R. Champagne-Hartley, G. A. Deeter, J. D. Campbell, B. Reck, D. Urban and M. F. Cunningham, Amphiphilic block copolymers as stabilizers in emulsion polymerization: effects of the stabilizing block molecular weight dispersity on stabilization performance, Macromolecules, 2015, 48, 8913.

3 E. Zuccatoa, S. Castiglioni and R. Fanelli, Identification of the pharmaceuticals for human use contaminating the Italian aquatic environment, J. Hazard. Mater., 2005, 122, 205.

4 C. Zwiener, Occurrence and analysis of pharmaceuticals and their transformation products in drinking water treatment, Anal. Bioanal. Chem., 2007, 387, 1159.

5 N. Zhang, Y. R. Xu, Z. L. Li, C. R. Yan, K. Mei, M. L. Ding, S. C. Ding, P. Guan, L. W. Qian, C. B. Du and X. L. Hu, Molecularly Imprinted Materials for Selective Biological Recognition, Macromol. Rapid Commun., 2019, 40, 1900096.

6 W. J. Zhu, W. Ma, C. X. Li, J. M. Pan and X. H. Dai, Welldesigned multihollow magnetic imprinted microspheres based on cellulose nanocrystals (CNCs) stabilized Pickering double emulsion polymerization for selective adsorption of bifenthrin, Chem. Eng. J., 2015, 276, 249.

7 J. M. Pan, Y. J. Yin, M. Y. Gan, M. J. Meng, X. H. Dai, R. R. Wu, W. D. Shi and Y. S. Yan, Fabrication and evaluation of molecularly imprinted multi-hollow microspheres adsorbents with tunable inner pore structures derived from templating Pickering double emulsions, Chem. Eng. J., 2015, 266, 299.

8 J. D. Dai, X. Wei, Z. J. Cao, Z. P. Zhou, P. Yu, J. M. Pan, T. B. Zou, C. X. Li and Y. S. Yan, Highly-controllable imprinted polymer nanoshell at the surface of magnetic halloysite nanotubes for selective recognition and rapid adsorption of tetracycline, RSC Adv., 2014, 4, 7967.

9 S. C. Ding, Z. L. Li, Y. Cheng, C. B. Du, J. F. Gao, Y. W. Zhang, N. Zhang, Z. T. Li, N. H. Chang and X. L. Hu, Enhancing adsorption capacity while maintaining specific recognition performance of mesoporous silica: a novel imprinting strategy with amphiphilic ionic liquid as surfactant, Nanotechnology, 2018, 29, 375604.

10 J. J. Yang, Y. Li, J. C. Wang, X. L. Sun, R. Cao, H. Sun, C. N. Huang and J. P. Chen, Molecularly imprinted polymer microspheres prepared by Pickering emulsion polymerization for selective solid-phase extraction of eight bisphenols from human urine samples, Anal. Chim. Acta, 2015, 872, 35.

11 B. P. Binks and S. O. Lumsdon, Influence of particle wettability on the type and stability of surfactant-free emulsions, Langmuir, 2000, 16, 8622.

12 Z. G. Cui, K. Z. Shi, Y. Z. Cui and B. P. Binks, Double phase inversion of emulsions stabilized by a mixture of $\mathrm{CaCO}_{3}$ nanoparticles and sodium dodecyl sulphate, Colloids Surf., A, 2008, 329, 67.

13 Z. Y. Lin, Z. Zhang, Y. M. Li and Y. L. Deng, Magnetic nano$\mathrm{Fe}_{3} \mathrm{O}_{4}$ stabilized Pickering emulsion liquid membrane for selective extraction and separation, Chem. Eng. J., 2016, 288, 305.

14 J. M. Pan, Y. J. Yin, Y. Ma, F. X. Qiu, Y. S. Yan, X. H. Niu, T. Zhang and $\mathrm{X}$. Bai, Hierarchical porous molecule/ion imprinted polymers with double specific binding sites: Combination of Pickering HIPEs template and pore-filled strategy, Chem. Eng. J., 2016, 301, 210.

15 S. Stiller, H. Gers-Barlag, M. Lergenmueller, F. Pflücker, J. Schulz, K. P. Wittern and R. Daniels, Investigation of the stability in emulsions stabilized with different surface modified titanium dioxides, Colloids Surf., A, 2004, 232, 261.

16 N. P. Ashby and B. P. Binks, Pickering emulsions stabilised by Laponite clay particles, Phys. Chem. Chem. Phys., 2000, 2, 5640 .

17 C. M. Li, J. J. Tan, J. W. Gu, L. Qiao, B. L. Zhang and Q. Y. Zhang, Rapid and efficient synthesis of isocyanate microcapsules via thiol-ene photopolymerization in Pickering emulsion and its application in self-healing coating, Compos. Sci. Technol., 2016, 123, 250.

18 Z. M. Gao, X. Q. Yang, N. N. Wu, L. J. Wang, J. M. Wang, J. Guo and S. W. Yin, Protein-based pickering emulsion and oil gel prepared by complexes of zein colloidal particles and stearate, J. Agric. Food Chem., 2014, 62, 2672.

19 Y. F. Zhu, Y. Zheng, L. Zong, F. Wang and A. Q. Wang, Fabrication of magnetic hydroxypropyl cellulose-gpoly(acrylic acid) porous spheres via Pickering high internal phase emulsion for removal of $\mathrm{Cu}^{2+}$ and $\mathrm{Cd}^{2+}$, Carbohydr. Polym., 2016, 149, 242.

20 H. Saari, K. Heravifar, M. Rayner, M. Wahlgren and M. Sjöö, Preparation and characterization of Starch particles for use in Pickering emulsions, Cereal Chem., 2016, 93, 116.

21 Y. Zhou, Z. Q. Qu, Y. S. Zeng, Y. K. Lin, Y. Li, P. Chung, R. Wong and U. Hagg, Neuroprotective effect of preadministration with Ganoderma lucidum spore on rat hippocampus, Exp. Toxicol. Pathol., 2012, 64, 673.

22 X. Wang, X. L. Chen, Z. M. Qi, X. C. Liu, W. Z. Li and S. Y. Wang, A study of Ganoderma lucidum spores by FTIR microspectroscopy, Spectrochim. Acta, Part A, 2012, 91, 285.

23 D. Zhao, J. S. Li, W. Suen, M. W. Chang and J. Huang, Preparation and characterization of Ganoderma lucidum spores-loaded alginate microspheres by electrospraying, Mater. Sci. Eng., C, 2016, 62, 835.

24 L. L. Fan, Y. Wang, M. Zhao, J. Z. Song, J. Y. Wang and Z. J. Jin, Magnetic ganoderma lucidum spore microspheres: a novel material to immobilize CotA 
multicopper oxidase for dye decolorization, J. Hazard. Mater., 2016, 313, 122.

25 O. u. Rahman, S. Shi, J. H. Ding, D. Wang, S. Ahmad and H. B. Yu, Lignin nanoparticles: Synthesis, characterization and their corrosion protection performance, New J. Chem., 2018, 42, 3415.

26 J. M. Pan, Q. Qu, J. Cao, D. Yan, J. X. Liu, X. H. Dai and Y. S. Yan, Molecularly imprinted polymer foams with welldefined open-cell structure derived from Pickering HIPEs and their enhanced recognition of $\lambda$-cyhalothrin, Chem. Eng. J., 2014, 253, 138.

27 J. M. Pan, J. Zeng, Q. Cao, H. P. Gao, Y. Gen, Y. C. Peng, X. H. Dai and Y. S. Yan, Hierarchical macro and mesoporous foams synthesized by HIPEs template and interface grafted route for simultaneous removal of $\lambda$ cyhalothrin and copper ions, Chem. Eng. J., 2016, 284, 1361.

28 L. Y. Geng, X. Kou, J. D. Lei, H. J. Su, G. H. Ma and Z. G. Su, Preparation, characterization and adsorption performance of molecularly imprinted microspheres for erythromycin using suspension polymerization, J. Chem. Technol. Biotechnol., 2012, 87, 635.

29 T. Renkecz, G. Mistlberger, M. Pawlak, V. Horvath and E. Bakker, Molecularly imprinted polymer microspheres containing photoswitchable spiropyran-based binding sites, ACS Appl. Mater. Interfaces, 2013, 5, 8537.

30 B. P. Binks, J. H. Clint, G. Mackenzie, C. Simcock and C. P. Whitby, Naturally occurring spore particles at planar fluid interfaces and in emulsions, Langmuir, 2005, 21, 8161.

31 H. W. Duan, D. Y. Wang, D. G. Kurth and H. Möhwald, Directing self-assembly of nanoparticles at water/oil interfaces, Angew. Chem., 2004, 116, 5757.

32 C. Giovannoli, C. Passini, L. Anfossi, F. D. Nardo, G. Spano, V. Maurino and C. Baggiani, Comparison of binding behavior for molecularly imprinted polymers prepared by hierarchical imprinting or Pickering emulsion polymerization, J. Sep. Sci., 2015, 38, 3661.

33 T. Fan, W. M. Yang and N. W. Wang, Molecularly imprinted polymer microspheres derived from Pickering emulsions polymerization in determination of di(2-ethylhexyl) phthalate in bottled water samples, J. Appl. Polym. Sci., 2016, 133, 43484.

34 J. J. Ma, Z. Y. Fu, P. Y. Ma, Y. L. Su and Q. J. Zhang, Breaking and characteristics of Ganoderma lucidum spores by high speed entrifugal shearing pulverizer, J. Wuhan Univ. Technol., Mater. Sci. Ed., 2007, 22, 617.

35 Y. J. Fu, W. Liu, Y. G. Zu, X. G. Shi, Z. G. Liu, G. Schwarz and T. Efferth, Breaking the spores of the fungus Ganoderma lucidum by supercritical $\mathrm{CO}_{2}$, Food Chem., 2009, 112, 71 .
36 W. J. Zhu, W. Ma, C. X. Li, J. M. Pan, X. H. Dai, M. Y. Gan, Q. Qu and Y. L. Zhang, Magnetic molecularly imprinted microspheres via yeast stabilized Pickering emulsion polymerization for selective recognition of $\lambda$-cyhalothrin, Colloids Surf., A, 2014, 453, 27.

37 E. M. Saad, A. Madbouly, N. Ayoub and R. M. El Nashar, Preparation and application of molecularly imprinted polymer for isolation of chicoric acid from Chicorium intybus L. medicinal plant, Anal. Chim. Acta, 2015, 877, 80.

38 Y. J. Yin, J. M. Pan, J. Cao, Y. Ma, G. Q. Pan, R. R. Wu, X. H. Dai, M. J. Meng and Y. S. Yan, Rationally designed hybrid molecularly imprinted polymer foam for highly efficient $\lambda$-cyhalothrin recognition and uptake via twice imprinting strategy, Chem. Eng. J., 2016, 286, 485.

39 H. K. Chung, W. H. Kim, J. Park, J. Cho, T. Y. Jeong and P. K. Park, Application of Langmuir and Freundlich isotherms to predict adsorbate removal efficiency or required amount of adsorbent, J. Ind. Eng. Chem., 2015, 28, 241.

40 S. C. Liu, J. M. Pan, H. J. Zhu, G. Q. Pan, F. X. Qiu, M. J. Meng, J. T. Yao and D. Yuan, Graphene oxide based molecularly imprinted polymers with double recognition abilities: The combination of covalent boronic acid and traditional noncovalent monomers, Chem. Eng. J., 2016, 290, 220.

41 J. X. Wang, J. M. Pan, Y. J. Yin, R. R. Wu, X. H. Dai, J. D. Dai, L. Gao and H. X. Ou, Thermo-responsive and magnetic molecularly imprinted $\mathrm{Fe}_{3} \mathrm{O}_{4}$ @carbon nanospheres for selective adsorption and controlled release of 2,4,5trichlorophenol, J. Ind. Eng. Chem., 2015, 25, 321.

42 Z. J. Wu, H. Joo and K. Lee, Kinetics and thermodynamics of the organic dye adsorption on the mesoporous hybrid xerogel, Chem. Eng. J., 2005, 112, 227.

43 R. Rakhshaee, M. Khosravi and M. T. Ganji, Kinetic modeling and thermodynamic study to remove $\mathrm{Pb}(\mathrm{II})$, Cd(II), Ni(II) and Zn(II) from aqueous solution using dead and living Azolla filiculoides, J. Hazard. Mater., 2006, 134, 120.

44 Y. Z. Zhu, T. W. Xue, J. M. Pan, X. Wei, J. D. Dai, L. Gao and Y. S. Yan, Facile synthesis of eggshell-stabilized erythromycin-based imprinted composites for recognition and separation applications, $R S C A d v ., 2015,5,89030$.

45 J. Liu, D. Z. Yin, S. Zhang, H. Liu and Q. Y. Zhang, Synthesis of polymeric core/shell microspheres with spherical viruslike surface morphology by Pickering emulsion, Colloids Surf., A, 2015, 466, 174. 